\title{
Creation of a multiaccess database for hepatopancreaticobiliary surgery using open-source technology in a country that lacks electronic clinical database management systems
}

\author{
Malaka JAYAWARDENE*, Malith Sachintha NANDASENA, Upul DE SILVA, Aloka PATHIRANA
}

Hepatopancreaticobiliary Unit - Professorial Surgical Unit, Colombo South Teaching Hospital, Dehiwala-Mount Lavinia, Sri Lanka

Introduction: State sector hospitals in Sri Lanka lack electronic database management systems. The database at the HPB unit at Colombo South Teaching Hospital was based on a rudimentary Google-Sheet that wasn't maintenance-friendly, prone to inconsistencies, and lacked data retrievability for analysis purposes.

Methods: Using cloud-based Google services and AppSheet, a multiaccess mobile app was developed to store HPB data. The author spent 2 months studying the web platform to create a password-protected app. Consent was obtained from patients to maintain clinical data through the app using mobile devices of the HPB team members. After 25 months of use of the app, this abstract analyses the overall data.

Results: The app can record 254 data variables per patient, of which 222 are analyzable. The database has so far 1,561 patients referred to and managed at the HPB unit since November 2018 in which 566 liver (M:F 2:1), 578 pancreatic (M:F 1.5:1), and 417 biliary pathologies (M:F 1:1.2) have been diagnosed. 857 have malignant pathologies, 523 have benign pathologies. 455 had a conclusive surgical management decision, 477 had a nonsurgical management decision. $74 \%(\mathrm{n}=420)$ of liver, 37\% $(\mathrm{n}=214)$ of pancreatic, and $53 \%(\mathrm{n}=$ 222) of biliary patients had cancers. Data on 239 HCC, 64 CRLM, 137 pancreatic adenocarcinomas, 16 pancreatic cystic neoplasms, 141 cholangiocarcinoma, and 43 gallbladder cancers are available in the database. In whom a management decision was reached, $31 \%$ of liver, $39 \%$ of pancreatic, and $49 \%$ of biliary patients were operated on.

Conclusions: This cost-free user-friendly solution can revolutionize database management for a low-income country. 